\title{
Theoretical Prediction of CNT-CF/PP Composite Tensile Properties Using Various Numerical Modeling Methods.
}

\begin{abstract}
Development of effective models to predict tensile properties of 'carbon nanotube coated carbon fibre reinforced polypropylene (CNT-CF/PP)' composites is briefly discussed. The composite taken as the reference is based on the highest growth mechanism of CNTs over carbon fibres. Halpin-Tsai and Combined Voigt-Reuss model has been implemented. Young's modulus for CNT-CF/PP composites has been found $4.5368 \mathrm{GPa}$ and the tensile strength has been estimated $45.367 \mathrm{MPa}$ considering the optimum operating condition of chemical vapor deposition (CVD) technique. Stiffness of the composite is represented through the stress-strain plots; stiffness is proportional to the steepness of the slope. There are slight deviations of results that have been found theoretically over the experimental issues.
\end{abstract}

Keyword: Carbon nanotube; Hierarchical composites; CNT-CF/PP; Tensile properties; Stiffness 